\title{
Model of the synchronization network functioning process in the context of intellectualization of network control functions
}

\author{
A.K. Kanaev ${ }^{1, *}$, E.V. Oparin ${ }^{2}$, and E.V. Oparina ${ }^{1}$ \\ ${ }^{1}$ Emperor Alexander I St. Petersburg state transport university (PGUPS) 190031, 9 Moskovsky pr., \\ Saint Petersburg, Russia \\ ${ }^{2}$ Institute for the design of signaling, interlocking, communication and radio in railway transport \\ Giprotransignalsvyaz - branch of Roszheldorproject, 192007, st. Borovaya, 49, St. Petersburg, Russia
}

\begin{abstract}
This paper provides a simulation model of the synchronization network (SN) functioning process, which is distinguished by the completeness of accounting for the functioning states. The key indicator of the synchronization network functioning process is the duration of the SN control cycle, which is understood as the time from the moment a failure occurs on the synchronization network until the moment the synchronization signal transmission mode is restored. On the basis of the developed simulation model of the synchronization network functioning process, the duration of the $\mathrm{SN}$ control cycle is estimated depending on the time spent in individual states of the $\mathrm{SN}$ functioning process and on the characteristics of individual subsystems of the synchronization system. The results obtained can serve as a basis for developing sound strategies and plans for controlling the synchronization network.
\end{abstract}

\section{Introduction}

One of the most important subsystems of a telecommunication system (TCS) is a synchronization network, which performs the functions of generating, transmitting and distributing synchronization signals necessary to maintain the functioning of all digital TCS devices and provide communication services of a given quality. Each SN element is characterized by many parameters reflecting its state, and the synchronization network is a large and complex telecommunication subsystem [1-7]. At the same time, information about the state of the synchronization network elements is often incomplete and unreliable, and the requirements for the promptness of making decisions on the control of the synchronization network are constantly increasing. These factors make it difficult for the maintenance personnel to make timely and sound decisions on the control of the synchronization network. The key indicator of the synchronization network functioning process is the duration of the control cycle, which is understood as the time from the moment a failure occurs on the

\footnotetext{
* Corresponding author: onapuh@mail.ru
} 
synchronization network until the moment when the synchronization signal transmission mode is restored. Taking into account the special importance of the synchronization network as part of the TCS, decision makers (DM) on the control of the SN tend to lower the value of this indicator, regardless of the expenditure of financial, material and human resources. However, often the decisions made do not lead to the desired results, and the allocated resources are wasted. Thus, the work aimed at modeling the synchronization network functioning process and assessing the duration of the control cycle of the synchronization network under various scenarios of control actions and the effects of destabilizing factors should be considered relevant.

\section{Description of the synchronization network functioning process}

To form a model of the synchronization network functioning process, it is necessary firstly to consider the factors contributing to the transition of the synchronization network and its elements into a state of failure, the main elements of the synchronization network subject to the destructive effect of these factors and their characteristics; procedures for diagnosing the state of synchronization network elements with further consideration of measures to restore it [8-12].

The synchronization network functioning process in the TCS can be characterized by a set consisting of a finite set of states $\operatorname{Si}(i=\overline{1, n})$, and a set of residence times in each specific state $\operatorname{Ti}(i=\overline{1, \mathrm{n}})$.

In the general case, the set of states of the synchronization network functioning process consists of the following elements $\mathrm{Si}(i=\overline{1,11})$, and the specified process is shown in Fig. $1[8-12]:$

$\mathrm{S} 1 \mathrm{~b}$ - state of switching on and checking the operability of the synchronization network elements;

$\mathrm{S} 2 \mathrm{~b}$ - state of checking the synchronization network configuration for compliance with the architecture requirements;

$\mathrm{S} 3 \mathrm{~b}$ - state of setting, adjusting and establishing the mode of synchronous interaction of synchronization network elements;

$\mathrm{S} 4 \mathrm{~b}$ - synchronization provision state to which sync transmission corresponds;

$\mathrm{S} 5 \mathrm{p}$ - state of selecting a host and enabling an alternative primary synchronization source;

S6p - state of diagnostics of the synchronization network element by the built-in means of technical diagnostic (MTD) and restoration of the synchronization network element in accordance with the diagnostic results;

S7p - state of formation of a connected version of the structure of the synchronization network with a minimum number of switchings and restoration of the synchronization network;

$\mathrm{S} 8 \mathrm{~b}$ - state of restoration of the initial structure of the synchronization network with the restored element;

S9p - state of diagnostics of the synchronization network element by external MTD and restoration of the $\mathrm{SN}$ element in accordance with the diagnostic results;

$\mathrm{S} 10 \mathrm{p}$ - state of rebuilding the synchronization network with the structure optimal by the integral quality criterion;

$\mathrm{S} 11 \mathrm{~b}$ - state of the synchronization network decommissioning.

It is advisable to classify these states from the point of view of the decision-making subprocesses they contain. 
In accordance with the given set of states, the state $\mathrm{Si}$ with the index "b" corresponds to a state that assumes the execution of operations that do not require access to the control system (CS) of the synchronization network and making decisions on a set of alternatives. The state Si with the index " $p$ " corresponds to a state that presupposes access to the SN CS, the transfer of initial data and the formation of a rational solution on a significant set of alternatives in the SN SC. These states are also marked on the block diagram of the processes of functioning and restoration of the synchronization network (Fig. 1).

The processes of transitions of the synchronization network from one state Si to another state $\mathrm{Sj}$, as well as the process of its recovery in the event of failures, significantly depend on the activities of the maintenance personnel who are obliged to monitor the parameters of the synchronization network and quickly respond when these parameters go beyond the required standards; on the process of functioning of the diagnostic system as part of the technical operation system (TOS), which ensures the control of the diagnosed parameters with a given accuracy; on the subsystems of the control system that provide solutions for maintaining the synchronization network in a given state.

The state S4b corresponds to the stage of providing the synchronization mode. In other states, the synchronization network is partially inoperative or operates with the values of the parameters characterizing the quality of synchronization below the allowable ones. In these states, the maintenance personnel are engaged in identifying the reasons for the violation of the synchronization mode, carrying out measurements using built-in and external diagnostic tools (states S6p and S9p), changing topologies and synchronization schemes (states S5p, $\mathrm{S} 7 \mathrm{p}, \mathrm{S} 8 \mathrm{~b}, \mathrm{~S} 10 \mathrm{p}$ ), regulating and setting synchronization modes corresponding to the established norms (states S1b, S2b, S3b).

The synchronization network stays in each of the listed states for a certain time, which is characterized by a plurality of residence times $\mathrm{Ti}(i=\overline{1,11})$ in a particular state.

Maintenance personnel are informed about the values of the parameters of the synchronization network and its elements by measuring these parameters by means of diagnostics (MD). Taking into account the fact that an element of the synchronization network can be serviceable (operable) or malfunctioning at any given moment of time, as well as the fact that there is an error in the functioning of MD, the following measurement results are possible [1-7]:

- An efficient element of the synchronization network is recognized as operable;

- An inoperative element of the synchronization network is recognized as inoperative;

- An efficient element of the synchronization network was recognized as inoperative (type I error);

- An inoperative element of the synchronization network was recognized as operable (type II error).

The first option corresponds to the best case in which the process of functioning is not interrupted. In the second case, the synchronization network functioning process can pass from the mode of the quality category "a" to the mode of the quality category " $b$ " with the subsequent transition to the mode of the quality category "c" due to the need to restore the faulty element.

In the third and fourth cases, the recovery time increases due to an incorrect decision about the state of the synchronization network element. The third option is typical for cases of elimination of "false failures", and in the fourth, attempts are made to operate the faulty element until the true cause of the fault is clarified. 


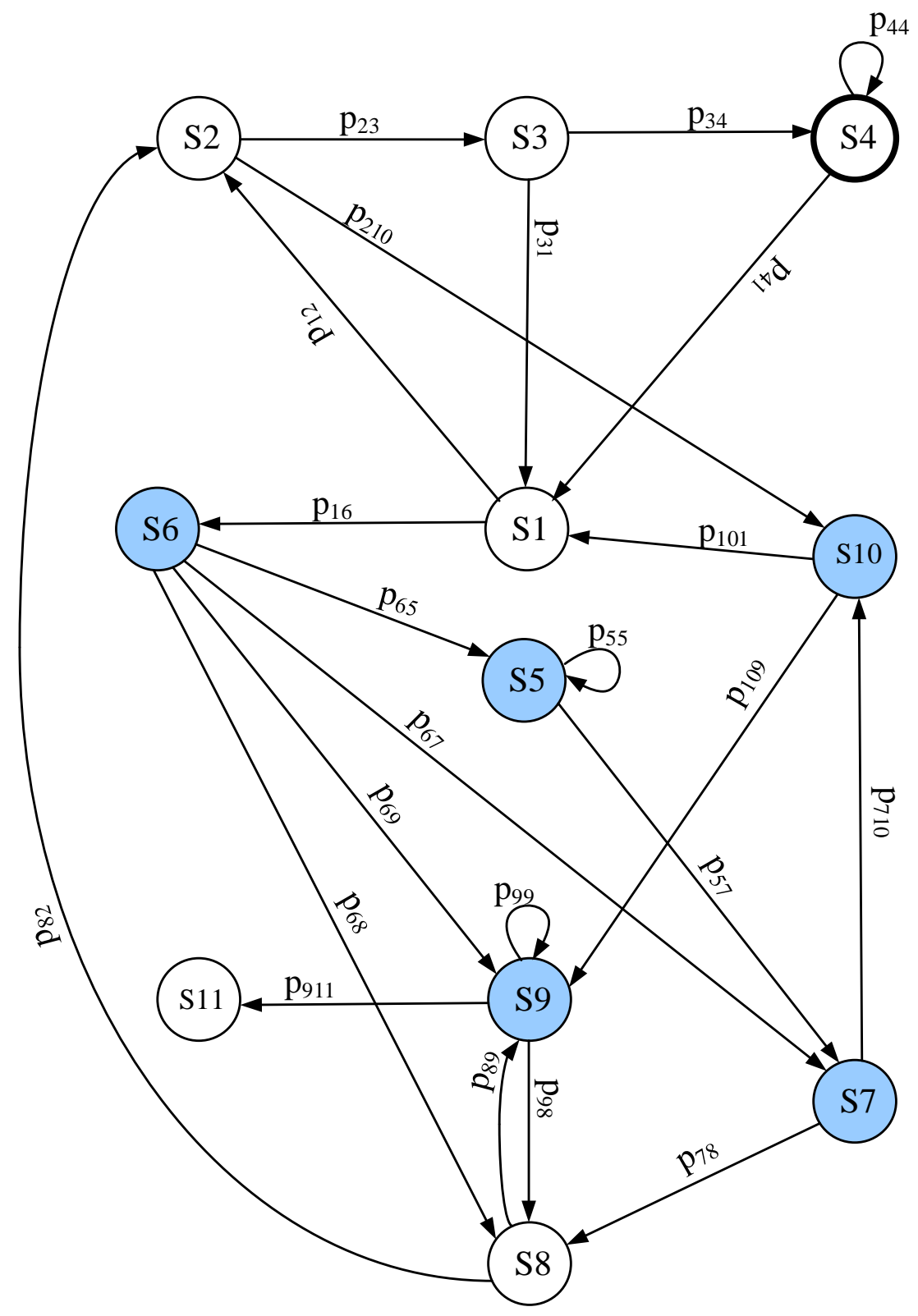

Fig. 1. The synchronization network functioning process.

Taking into account the particular importance of states in the sense of the time required to develop a solution in the control system by the synchronization network, which can be many times greater than the time for technological operations to restore the synchronization network, which involve accessing the control system, the problem of justified estimation of the CS resource consumption by the synchronization network, depending on the requirements to the duration of the synchronization network control cycle, is relevant.

It can be stated that the decision-making process is complex and multi-stage with a large number of cycles. This means that the time required for the formation of the solution cannot 
be neglected, since it can be comparable to the duration of the technological process of restoring the synchronization network, and in some cases, it can significantly exceed it.

\section{Simulation modeling of the synchronization network functioning process}

To study the synchronization network functioning process, a simulation model was built in the AnyLogic environment [13].

The AnyLogic simulation environment has a number of advantages, such as visibility of building a model, a convenient interface for entering and adjusting initial data, the ability to use various mechanisms for displaying simulation results with the ability to compare them [13].

The constructed simulation model looks as follows (Fig. 2).

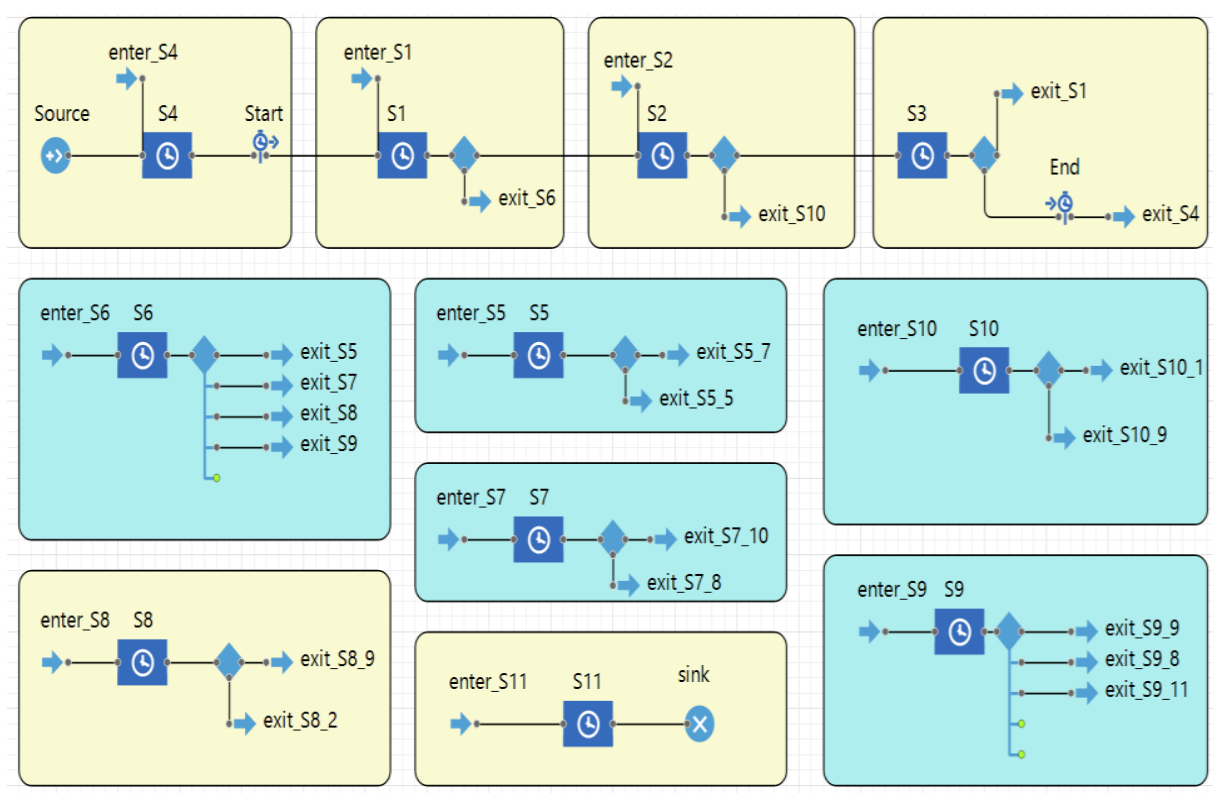

Fig. 2. Simulation model of the synchronization network functioning process.

The initial data for the simulation is shown in Table 1.

The initial data are taken from the practice of operating synchronization networks. The time spent in the S4 state corresponds to the coefficient of availability of the synchronization network $K_{A}=0.99999$. The rest of the times and probabilities of transitions from states to states correspond to the typical values of the performance indicators of the synchronization network and the control system of the synchronization network. This model makes it possible to estimate the duration of the control cycle of the synchronization network in states that are characterized by the values of the characteristics of the functioning process that differ from the standard values. For this purpose, a time counter was introduced into the simulation model, which makes it possible to track the values of the duration of the control cycle when the synchronization network functioning process leaves the S4 state, i.e. when a failure occurs, and returns to the $\mathrm{S} 4$ state, when the failure was eliminated as a result of the activities of the SN CS and TOS of SN. The simulation results are shown in Fig. 3.

Table 1. Initial data for simulation modeling. 


\begin{tabular}{|c|c|c|c|c|c|}
\hline No. & State name & & ignation & Value & Meas. \\
\hline 1. & $\begin{array}{l}\text { The state of switching on and checking } \\
\text { the efficiency of the elements of the } \\
\text { synchronization system }\end{array}$ & S1 & S1 & 0.5 & hour \\
\hline 2. & $\begin{array}{l}\text { The state of the network configuration } \\
\text { synchronization check for compliance } \\
\text { with the architecture }\end{array}$ & S2 & S2 & 1 & hour \\
\hline 3. & $\begin{array}{l}\text { The state of setting, adjusting and } \\
\text { establishing the mode of synchronous } \\
\text { interaction of synchronization network } \\
\text { elements }\end{array}$ & S3 & (Q) & 0.5 & hour \\
\hline 4. & $\begin{array}{l}\text { Synchronization provision state to } \\
\text { which sync transmission corresponds }\end{array}$ & S4 & S4 & 8640 & hour \\
\hline 5. & $\begin{array}{l}\text { The state of selecting a host and } \\
\text { enabling an alternative primary } \\
\text { synchronization source }\end{array}$ & S5 & S5 & 2 & hour \\
\hline 6. & $\begin{array}{l}\text { The state of diagnostics of the } \\
\text { synchronization network element by } \\
\text { the built-in means of technical } \\
\text { diagnostic (MTD) and restoration of } \\
\text { the synchronization network element in } \\
\text { accordance with the diagnostic results }\end{array}$ & S6 & 56 & 1 & hour \\
\hline 7. & $\begin{array}{l}\text { The state of formation of a connected } \\
\text { version of the structure of the } \\
\text { synchronization network with a } \\
\text { minimum number of switchings and } \\
\text { restoration of the synchronization } \\
\text { network }\end{array}$ & S7 & 57 & 4 & hour \\
\hline 8. & $\begin{array}{l}\text { The state of restoration of the initial } \\
\text { structure of the synchronization } \\
\text { network with the restored element }\end{array}$ & S8 & S8 & 3 & hour \\
\hline 9. & $\begin{array}{l}\text { The state of diagnostics of the } \\
\text { synchronization network element by } \\
\text { external MTD and restoration of the SN } \\
\text { element in accordance with the } \\
\text { diagnostic results }\end{array}$ & S9 & (1) & 5 & hour \\
\hline 10. & $\begin{array}{l}\text { The state of rebuilding the } \\
\text { synchronization network with the } \\
\text { structure optimal by the integral quality } \\
\text { criterion }\end{array}$ & S10 & $\begin{array}{r}510 \\
\text { (Q) }\end{array}$ & 1 & hour \\
\hline 11. & $\begin{array}{l}\text { The state of the synchronization } \\
\text { network decommissioning }\end{array}$ & S11 & S11 & - & - \\
\hline
\end{tabular}

The matrix of transition probabilities from one state to another has the following form (1). 


$$
P=\left(\begin{array}{ccccccccccc}
0 & 0.95 & 0 & 0 & 0 & 0.05 & 0 & 0 & 0 & 0 & 0 \\
0 & 0 & 0.95 & 0 & 0 & 0 & 0 & 0 & 0 & 0.05 & 0 \\
0.05 & 0 & 0 & 0.95 & 0 & 0 & 0 & 0 & 0 & 0 & 0 \\
1 & 0 & 0 & 0 & 0 & 0 & 0 & 0 & 0 & 0 & 0 \\
0 & 0 & 0 & 0 & 0.5 & 0 & 0.5 & 0 & 0 & 0 & 0 \\
0 & 0 & 0 & 0 & 0.25 & 0 & 0.25 & 0.25 & 0.25 & 0 & 0 \\
0 & 0 & 0 & 0 & 0 & 0 & 0 & 0.95 & 0 & 0.05 & 0 \\
0 & 0.95 & 0 & 0 & 0 & 0 & 0 & 0 & 0.05 & 0 & 0 \\
0 & 0 & 0 & 0 & 0 & 0 & 0 & 0.9 & 0.05 & 0 & 0.05 \\
0.5 & 0 & 0 & 0 & 0 & 0 & 0 & 0 & 0.5 & 0 & 0 \\
0 & 0 & 0 & 0 & 0 & 0 & 0 & 0 & 0 & 0 & 0
\end{array}\right)
$$

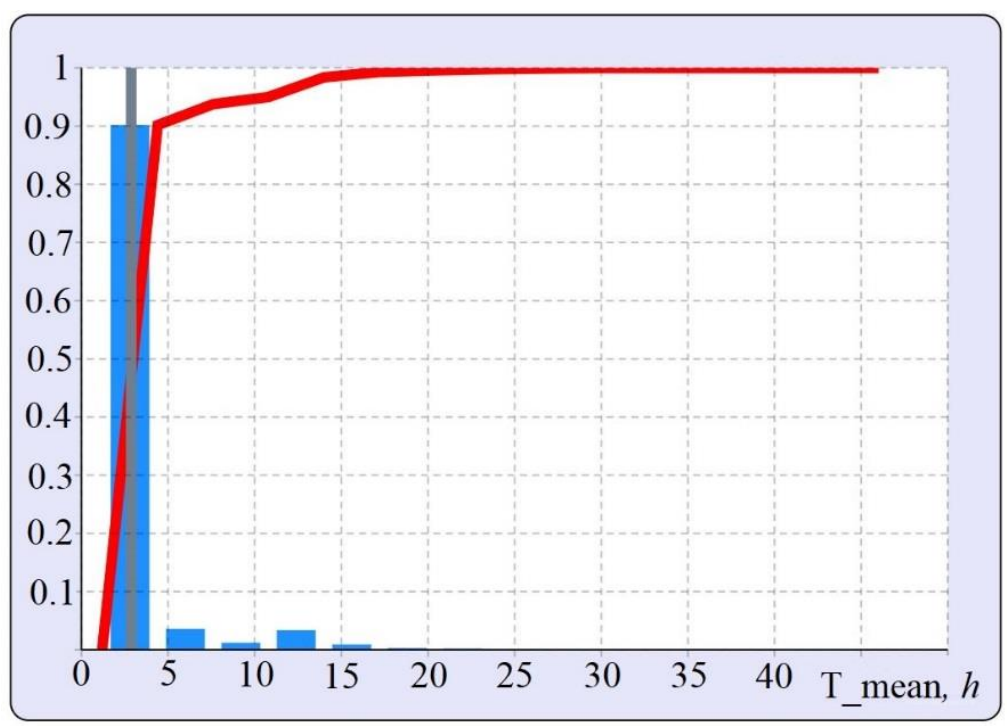

Fig. 3. Histogram of the duration of the synchronization network control cycle.

The simulation results show that the average duration of the synchronization network control cycle Tav is $2.92 \mathrm{~h}$. However, situations may arise where the control cycle time is significantly longer than this value.

\section{Assessment of the influence of individual states on the duration of the synchronization network control cycle}

Synchronization network control decision makers have a desire to shorten the control cycle time when failures occur. The financial and material resources at the disposal of the SN CS and the decision maker can be distributed in different ways between the states to perform the task of restoring the synchronization network. Taking into account the limited financial and material resources, decision makers strive to develop such a strategy for spending resources so that the duration of the synchronization network control cycle is minimal.

The constructed simulation model makes it possible to estimate the average duration of the synchronization network control cycle Tav, depending on the duration of the synchronization network functioning process in a separate state. Note that reducing the time spent in a particular state is usually associated with a more intensive expenditure of resources for this state. 
Thus, the decision maker can distribute the available resources for solving diverse tasks for individual states in accordance with the chosen strategy for reducing the duration of the synchronization network control cycle. The simulation results are shown in Fig. 4 - 8.
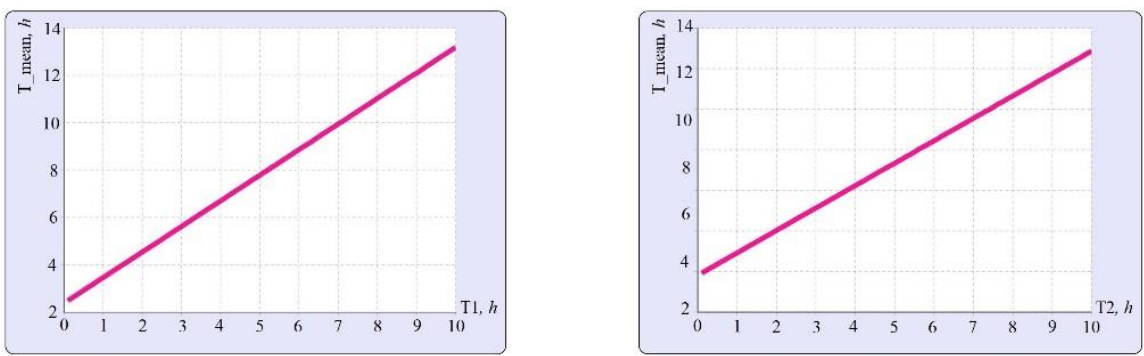

Fig. 4. Dependence of the average duration of the synchronization network control cycle on the duration of being in states S1 and S2.
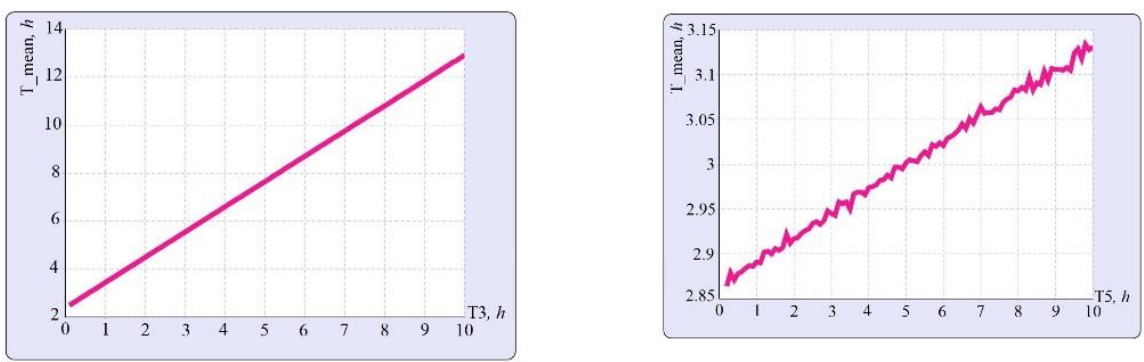

Fig. 5. Dependence of the average duration of the synchronization network control cycle on the duration of being in states S3 and S5.
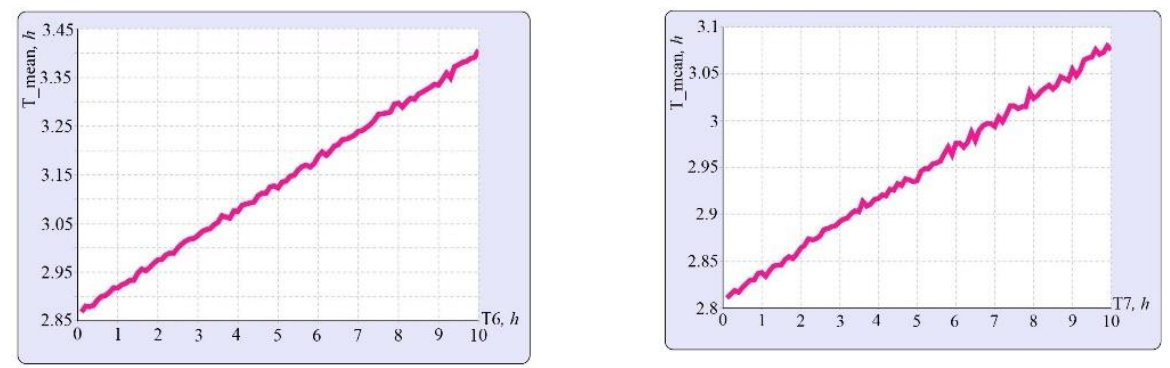

Fig. 6. Dependence of the average duration of the synchronization network control cycle on the duration of being in states S6 and S7.
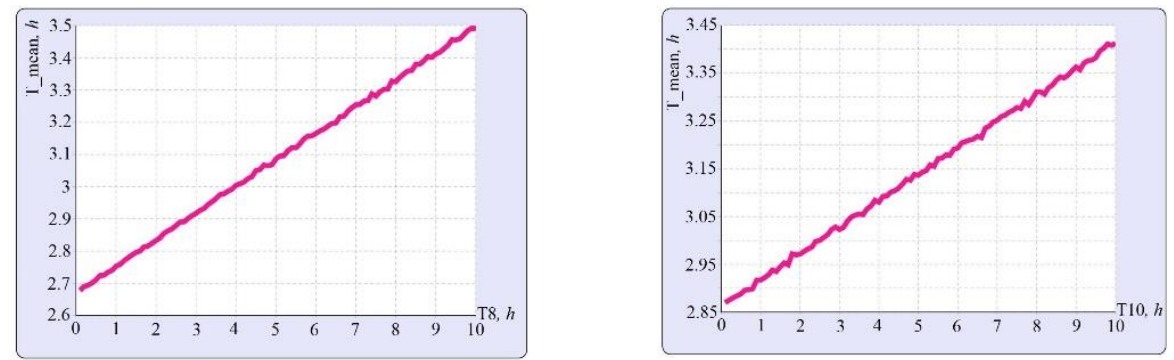
Fig. 7. Dependence of the average duration of the synchronization network control cycle on the duration of being in states $\mathrm{S} 8$ and $\mathrm{S} 9$.

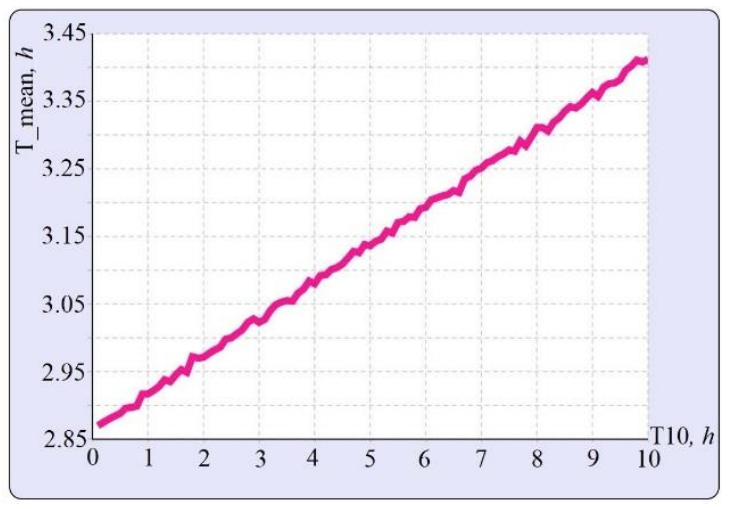

Fig. 8. Dependence of the average duration of the synchronization network control cycle on the duration of being in states S10.

The simulation results show that in accordance with the given initial data according to Table 1 and the matrix of transition probabilities (1), the duration of the control cycle significantly depends on the time spent in the states S1, S2 and S3, and weakly depends on the stay in other states. Considering these results, the decision maker can most efficiently use a significant part of the resources at those moments of time when the synchronization network functioning process is in states S1, S2 and S3, since reducing the time spent in states S1, S2 and S3 will significantly affect the reduction of the entire duration of the synchronization network control cycle. Nevertheless, despite the results obtained, decision makers should not neglect the rest of the states, because they are also key in the process of restoring the synchronization network functioning process. Having certain requirements for the duration of the synchronization network control cycle, the decision maker can choose an appropriate strategy for spending resources to restore the synchronization network functioning process.

\section{Assessment of the influence of the network synchronization subsystem characteristics on the duration of the synchronization network control cycle}

A significant role in the duration of the synchronization network control cycle is influenced by the characteristics of the synchronization network itself, the technical operation system of the SN and the SN CS. This influence is expressed in the values of the transition probabilities between the states of the synchronization network functioning process.

High values of the efficiency and validity of decision-making in the SN CS, the values of the rate of collection and processing of diagnostic information in the technical operation system are usually characteristic of increased values of transient probabilities associated with the restoration of the synchronization network. On the contrary, low values of the key characteristics of the SN CS and technical operation system of the SN are characteristic for the increased values of transient probabilities associated with failures of the synchronization network.

The presented simulation model of the synchronization network functioning process (Fig. 2) makes it possible to track the relationship between the values of the transition probabilities 
and the duration of the SN control cycle. Examples of these relationships are shown in Fig. $9-10$.
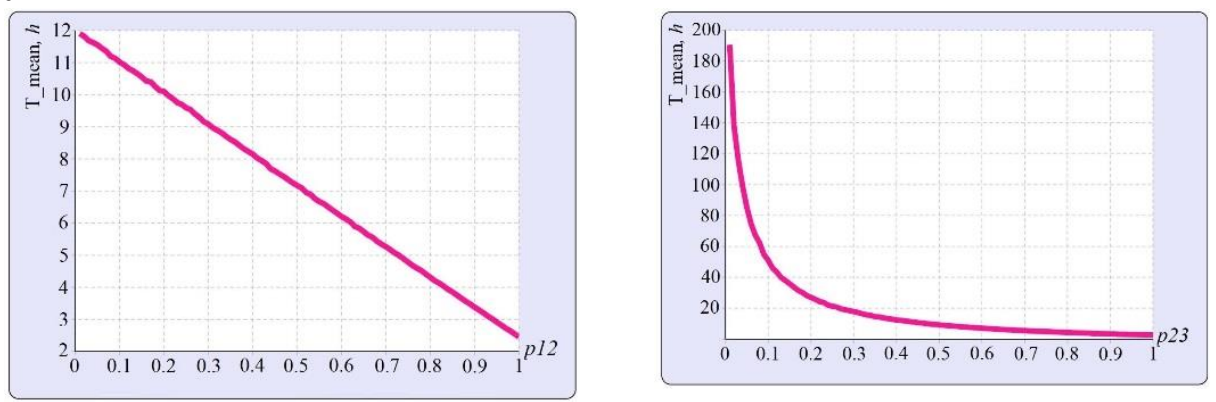

Fig. 9. Dependence of the average duration of the synchronization network control cycle on the probability of transitions $\mathrm{p} 12$ and $\mathrm{p} 23$.

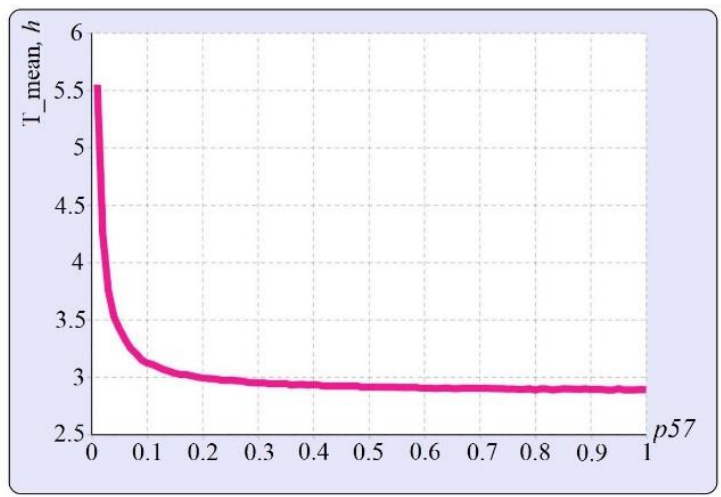

Fig. 10. Dependence of the average duration of the synchronization network control cycle on the transition probability p57.

These relationships, based on the requirements for the duration of the synchronization network control cycle, can serve as a basis for changing the technical characteristics of individual synchronization subsystems. By upgrading individual subsystems of the synchronization system, it is possible to achieve a change in the values of the transient probabilities, and, consequently, to bring the duration of the synchronization network control cycle to the standard values.

\section{Conclusion}

The synchronization network is an important TCN subsystem that directly affects the quality of communication services. In this connection, this paper presents the results of assessing the duration of the synchronization network control cycle depending on the individual states of the synchronization network functioning process and the characteristics of the synchronization system subsystems. Based on the analysis of the SN functioning process, the results obtained will make it possible for the decision-makers to control the synchronization network, reasonably allocate resources to restore the synchronization network functioning process and to modernize individual synchronization system.

It is assumed that the initial data for the simulation will be determined from the practice of operating a specific synchronization network, which will allow obtaining a model of the synchronization network functioning process with real properties [14-16]. This model is 
universal, is distinguished by the completeness of accounting of states and can be applied to any synchronization network, which allows, when regulatory requirements are imposed on the duration of the control cycle, forming a set of strategies and plans for controlling the synchronization network.

\section{References}

1. Sabella R, Testa F, Iovanna P, Bottari G 2014 IEEE Communication Magazine 35

2. Ferrant J-L, Ruffini S 2011 IEEE Communication Magazine 132

3. Hann K, Jobert S, Rodrigues S 2012 IEEE Communication Magazine 152

4. ITU-T Recommendation G.781: Synchronization layer functions for frequency synchronization based on the physical layer

5. ITU-T Recommendation G.8261 : Timing and synchronization aspects in packet networks

6. RFC 5905 Network Time Protocol Version 4: Protocol and Algorithms Specification

7. IEEE 1588 Precision Time Protocol (PTP)

8. Correia M, Sousa J, Combo Á, Rodrigues A P, Carvalho B B, Batista A J N, Gonçalves B, Correia C, Varandas C 2012 Implementation of IEEE-1588 timing and synchronization for ATCA control and data acquisition systems. - Fusion Engineering and Design 87(12) 2178

9. Yuhei Kikuya, Hideaki Ishii 2016 IFAC-PapersOnLine 49(22) 181

10. Carli R, Chiuso A, Schenato L, Zampieri S 2011 IEEE Trans. Autom. Control 561146

11. He J, Cheng P, Shi L, Chen J, Sun Y 2014 IEEE Trans. Autom. Control 59660

12. Kadowaki Y, Ishii H 2015 IEEE Trans. Autom. Control 602266

13. Website of Anylogic company https://www.anylogic.ru/

14. Schenato L, Fiorentin F 2011 Automatica 471878

15. Solis R, Borkar V, Kumar P R 2006 Proc. 45th IEEE Conf. on Decision and Control 2734

16. Wu Y C, Chaudhari Q, Serpedin E 2011 IEEE Signal Processing Mag. 47(1) 124 